\section{A. ARS BILDUMA ISSN 1989-9262 UPV/EHU Press} ARSBILDUMA (CC BY-NC-ND 4.0)

https://doi.org/10.1387/ars-bilduma.17200 BIBLID [(2019), 9; 155-167]

Recibido: 24/10/2016 Aceptado: 06/02/2017

\section{LEIRE MACAZAGA LANAS}

Universidad Internacional de La Rioja (UNIR)

Avenida de la Paz, 137

26006, Logroño (La Rioja)

leire.macazaga@unir.net

ORCID ID: 0000-0002-8121-6035

\title{
LA INCURSIÓN DE BONIFACIO ALFONSO EN EL GRABADO Y SU VINCULACIÓN CON LA DENOMINADA ESCUELA DE CUENCA
}

\author{
THE INCURSION OF BONIFACIO ALFONSO IN THE ENGRAVING AND ITS LINK \\ WITH THE SO CALLED SCHOOL OF CUENCA
}

L'INCURSION DE BONIFACIO ALFONSO DANS LA GRAVURE EL DE SES RELATIONS AVEC LES SOI-DISANT CUENCA ÉCOLE

\section{RESUMEN}

El presente artículo analiza la incursión de Bonifacio Alfonso en el grabado gracias a su relación con Fernando Zóbel y su vinculación al Museo de Arte Abstracto Español de Cuenca. En la ciudad conquense el artista vizcaíno tiene la oportunidad de adentrarse en esta disciplina imposible de practicar en el País Vasco debido a inexistencia de talleres para tal fin. Daremos cuenta de cómo se adentra en el grabado calcográfico así como de los diferentes talleres a los que acude para perfeccionar dichas técnicas llegando a convertirse en un auténtico artista-grabador.

\section{PALABRAS CLAVE}

Bonifacio Alfonso; obra gráfica; Escuela de Cuenca; década de los sesenta; década de los setenta.

\section{ABSTRACT}

The present article analyzes the incursion of Bonifacio Alfonso in the engraving thanks to its relation with Fernando and its link to the Spanish Abstract Art Museum of Cuenca. In the city of Cuenca the artist vizcaíno has the opportunity topenetrate into this discipline imposible practise in the Basque Country due to noexistence of workshops for such an end. We wil account for how it penetrates into the engranving calcográfico as well al for the different workshops to wich it comes to perfectthe above mentioned skills going so faras to turn into as authentic artista-engraver.

KEYWORDS

Bonifacio Alfonso; graphic work; Cuenca; sixties; seventies.

\section{RÉSUMÉ}

Le présent article trite de l'incursion de Bonifacio Alfonso dans la gravure, grâce à sa relation avec Fernando Zóbel et de ses liens avec le Musée d'Art Abstrait espagnolo de Cuenca. Dans la ville de Cuenca, làrtiste vizcaíno a la possibilité de s'aventurer dans cette discipline imposible d'exercer dans le Pays Basque en raison du manque d'ateliers dans ce but. Nous rendre compte de la façon dont il se projette dans les creux ainsi que les différents ateliers qui vont pourle perfectionnement de ces techniques, de devenir un véritable artiste-graveur.

\section{MOTS-CLÉS}

Bonifacio Alfonso; le travail graphique; Cuenca; une décennie de soixante; une décennie de soixante-dix. 


\section{INTRODUCCIÓN}

A lo largo de estas páginas se va a analizar los comienzos de Bonifacio Alfonso en la práctica del grabado, por lo que centraremos nuestra atención a finales de la década de los años sesenta y, particularmente, en los setenta. Comprobaremos cómo el encuentro casual con el artista y editor de obra gráfica Fernando Zóbel va a ser capital para adentrarse en dicha disciplina. Gracias a él se asienta en Cuenca, ciudad en la que debido a la apertura del Museo de Arte Abstracto Español, fundado por el propio Zóbel, y el taller que en torno al museo le instala a Antonio Lorenzo, el artista vasco tiene la oportunidad de adentrarse en una nueva disciplina imposible de practicarla en su comunidad al carecer de talleres para tal fin.

A todo ello se le debe añadir, tal y como comprobaremos a continuación, cómo el intenso clima artístico que se genera en la ciudad conquense durante estas décadas propicia una corriente de aire fresco con lo que supone de apertura a la vanguardia en un momento de gran agitación política, ya que nos situamos entre los últimos años de la dictadura franquista y la transición. Además de contar con la presencia de Zóbel, considerado como un verdadero mecenas, y el Museo de Arte Abstracto Español, también residen los artistas del grupo El Paso. Entre ellos, como analizaremos, se encuentra Antonio Saura quien, a igual que le sucede a Bonifacio, tiene la posibilidad de viajar al extranjero y mostrar las nuevas corrientes y tendencias que observa al resto de artistas que se encuentran en la ciudad.

\section{SUS INICIOS EN LA OBRA GRÁFICA. AÑOS DE FORMACIÓN EN DI- VERSOS TALLERES}

Bonifacio Alfonso Gómez (San Sebastián, 1933-2011) se introduce en el mundo del grabado en 1968 de la mano del polifacético Fernando Zóbel, quien le invita un verano a Cuenca para conocer el Museo de Arte Abstracto Español, inaugurado el 30 de junio de $1966^{1}$. Como comprobaremos más adelante, allí aprende la técnica calcográfica con

1 A pesar de que Fernando Zóbel (Manila, 1924 - Roma, 1984) conjuga múltiples facetas -pintor, grabador, coleccionista, historiador, profesor, bibliógrafo, mecenas, fundador de un museo, etc.- nos interesa fundamentalmente su vertiente como editor y difusor de obra gráfica. Tras tres años de arduo trabajo en 1966 ve cumplido su sueño de abrir en la ciudad de Cuenca un Museo que acoja una muestra de
Antonio Lorenzo y con el norteamericano Bernard Childs. Hay que esperar, por tanto, una década, desde que realiza su primera exposición individual de pintura en el Ateneo de Guipúzcoa en 1958, para que se adentre en el arte gráfico. Se debe tener en cuenta cómo debido a que tras el final de la contienda civil la enseñanza artística en el País Vasco es prácticamente inexistente, los que desean completar su formación artística deben sortear un sin fin de trabas. Aquellos que deciden probar suerte en el grabado deben buscar fuera talleres tanto nacionales como extranjeros que cuenten con equipos de calidad, con maestros-estampadores especializados en cada uno de los procedimientos, poniendo a su disposición tanto los materiales y herramientas como los conocimientos necesarios para su dominio ${ }^{2}$. panorama del arte actual. La idea se remonta unos años antes cuando en 1961 se establece en Madrid entabla amistad con un nutrido grupo de artistas abstractos como Antonio Lorenzo, Luís Feito, Eusebio Sempere, Gerardo Rueda y Gustavo Torner. Este último, es quien le invita, en 1963, a conocer su localidad natal, Cuenca. Ciudad que le cautiva desde el principio y en la cual decide instalar el Museo, del que Gustavo Torner es nombrado codirector y Gerardo Rueda conservador. Entre los artistas abstractos españoles que forman parte de los fondos del museo destacan Rueda, Sempere, Saura, Chirino, Lorenzo, Torner, Feito, Tàpies, Millares, Chillida, Oteiza o Mompó, entre otros. Para un mayor conocimiento de su trayectoria artística y así como del taller de grabado que inaugura en torno al Museo de Arte Abstracto Español de Cuenca se pueden consultar las siguientes publicaciones de MUSEO DE ARTE ESPAÑOL DE CUENCA: Colección de Arte Abstracto Español. Casas Colgadas. Museo Cuenca [Cat. Exp.], Museo de Arte Abstracto Español de Cuenca, 1966; ZÓBEL, F.: "Debe acabar la incertidumbre laboral del artista español", El País, Madrid, 8 de marzo, 1978; ORTUÑO, P.: "Crece el Museo de Arte

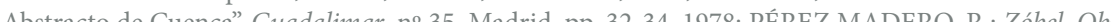
(g) grafica completa, Diputación Provincial de Cuenca. Departanento de Cultura, 199, DE LA TORRE, y DE LUCAS RAMIREZ, J.: La poética de Cuenca: 40 años después, 1964-2000 [Cat. Exp.], Centro Cultural de la Villa, Ayuntamiento de Madrid, 2004. MACAZAGA LANAS, L.: "Talleres de grabado en el País Vasco durante el tardo franquismo y la transición”, Aacadigital, n² 23, 2013. https://dialnet.unirioja. es/servlet/articulo?codigo=4530301 (consultado el 20/02/2016)

2 Para una mayor información sobre este tema se pueden consultar las siguientes publicaciones: ARÓSTEGUI BARBIER, J. DE: La pintura vizcaína de la posguerra. Del Grupo del Suizo a la Asociación Artística Vizcaina Bilbao, La Gran Enciclopedia Vasca, 1972. ARRIBAS, Ma J: 40 años de Arte Vasto (1937 1977). Histo ia J docus Pais Vasco: 1940-1980, Madrid, Akal, 1985 o MACAZAGA LANAS, L.: "El difícil panorama artístico al que debieron hacer frente los grabadores vascos durante el tardofranquismo y la transición", Aacadigi tal, $\mathrm{n}^{\circ} 27,2014$. http://www.aacadigital.com/contenido.php?idarticulo=974 (consultado el 21/02/2016). 
El artista donostiarra coincide con Zóbel en la primera exposición de su obra que se celebra en Bilbao y de la que se vende todo ${ }^{3}$. La muestra tiene lugar, del 17 de diciembre de 1966 al 4 de enero de 1967, en la Galería Grises, dirigida por José Luís Merino, quien hasta ese momento se ocupa de su obra. Merino le ofrece un estudio abuhardillado en el Casco Viejo bilbaíno donde poder pintar los ratos libres que le dejan sus ocupaciones en la Imprenta Industrial, trabajo que consigue gracias a que gana un concurso de murales para La Naval de Bilbao. A pesar de que no realiza su primer grabado hasta que acude a Cuenca, a través de los trabajos que desempeña como publicista tiene la oportunidad de instruirse en los dominios de los utensilios de las artes gráficas

Anteriormente, a la edad de 24 ó 25 años ya ha tenido contacto, en San Sebastián, con empresas de artes gráficas como la empresa Discos Columbia donde se ha encargado de dibujar las portadas discográficas. Tras varios años como dibujante proyectista en Artes Gráficas Nerecán, trabaja en Publicidad Eiffel y más tarde en Iongraf, ubicada en Andoain (Gipuzkoa), como encargado de la sección de dibujo.

La trayectoria vital de Bonifacio ha sido muy intensa. Su padre, miliciano de la compañía Máximo Gorki (Batallón de Rusia), es fusilado durante la Guerra Civil. Su madre junto a sus dos hijos - Bonifacio y su hermana mayor, melliza de otra que nació muerta- se ve obligada a marcharse a Vizcaya, de ahí a Cantabria y a Asturias para finalmente residir en Francia durante un año. A su regreso a San Sebastián debido a las penurias económica por las que atraviesan deben residir en la casa de La Misericordia. Con siete años, siendo monaguillo y corista de la Catedral del Buen Pastor, se inicia en la actividad pictórica. A partir de entonces, para ganarse el pan compaginará su afición a la pintura con múltiples y variados oficios: botones de hotel, pinche de cocina, aprendiz de herrero, ebanista lavandero, pescador de bajura, camarero, aprendiz de torero, limpia botas, pintor de brocha gorda, batería en un conjunto de jazz y publicista ${ }^{6}$.

Finalmente, a pesar de que consigue un puesto fijo como publicista, decide abandonar ese oficio porque no soporta estar sujeto a un horario que apenas le deja tiempo para pintar.

3 RUIZ QUINTANO, I.: Bonifacio. Madrid, Ediciones Turner, 1992, p. 41

Bonifacio trabaja como publicista a jornada completa con el fin de percibir un salario fijo todos los meses.

RUIZ QUINTANO, I.: Bonifacio. Op. cit, p. 4

6 Ibidem, p. 41
Por ese motivo, cuando Zóbel le propone ir a Cuenca para dedicarse exclusivamente a su actividad artística, no se lo piensa dos veces ${ }^{7}$. Éste acude a su apartamento para adquirirle unas obras -que piensa destinarlas a engrosar los fondos de su colección- y sugerirle que acuda a Cuenca para que conozca el entramado cultural que se está gestando alrededor del Museo $^{8}$.

Finalmente, el artista donostiarra permanece en la ciudad, junto a su segunda mujer Mari Carmen Flores, prácticamente tres décadas (de 1968 a 1996). En un primer momento, subsisten gracias a que Zóbel le consigue un contrato verbal con la madrileña Galería Juana Mordó de 8000 pesetas al mes ${ }^{9}$. Durante una temporada, hasta que se instala en su propia casa, ubicada en la Plaza del Trabuco, el matrimonio reside en casa de José Guerrero y posteriormente en el estudio que Gerardo Rueda le compra a Antonio Saura ${ }^{10}$.

Una vez instalado en la ciudad de la Hoz del Huécar conoce el taller de grabado que Zóbel le monta a Antonio Lorenzo, artista del que recientemente Bonifacio ha adquirido un aguafuerte. A pesar de que hasta la fecha no ha realizado ningún grabado por desconocimiento técnico, su interés por dicha disciplina se acrecienta cuando en una exposición celebrada en la Galería Grises descubre la obra gráfica de Antonio Lorenzo. Le gusta tanto lo que ve que decide adquirir un grabado que paga a plazos al propietario, José Luís Merino ${ }^{11}$.
Entrevista llevada a cabo por la autora de este texto a Bonifacio Alfonso en San Sebastián, el 7 de ener de 2009. Se le realizaron un total de 58 preguntas. El encuentro estuvo motivado por la realización de las tesis doctoral denominada "Los grabadores de las década de los años sesenta y setenta en el País Vasco" dirigida por Ignacio Díaz Balerdi y Edorta Kortadi, Universiad del País Vasco, 2013.
8 A lo largo de la segunda mitad de los años sesenta van llegando a la ciudad de la Hoz del Huécar di- versos artistas que se vinculan con el museo. Además de los que ya hace tiempo que residen ella -como los pintores Antonio Saura y Gustavo Torner; el ceramista Pedro Mercedes; el poeta Federico Muelas o el escritor Cesar González Ruano-, se les unen los pintores Antonio Lorenzo, Gerardo Rueda, Eusebio Sempere, José Guerrero, Manuel Hernández Mompó, Manolo Millares, Abel Martín, Ángel Cruz, Sal- vador Victoria; el escultor Amadeo Gabino; los fotógrafos Fernando Nuño y Jaume Blassi y el ingeniero y coleccionista de arte abstracto, Mario Barbeŕ́; entre otros. Informán extráda de DE LA TORRE, A.: La poética de Cuenca: 40 años después, 1964-2000.Op. cit, p. 57.
9 RUIZ QUINTANO, I.: op. cit., p. 56
10 Entrevista.
11 Ibid.


Junto a Antonio Lorenzo aprende la técnica del grabado calcográfico. Graban juntos tres años hasta que reúne el dinero suficiente para comprarse una prensa en Madrid -la traslada en piezas- con la que puede estampar sus propias obras. Bonifacio admite que todo lo que sabe de grabado es gracias a Antonio Lorenzo ${ }^{12}$ quien tuvo con él una paciencia infinita ${ }^{13}$ En ese tiempo, perfecciona la técnica junto al grabador estadounidense de origen ruso Bernard Childs, a quien Zóbel -con el que durante esos años mantiene una abundante correspondencia- invita a conocer la ciudad. Finalmente la estancia se prolonga durante tres meses. Allí también tiene la oportunidad de relacionarse con los artistas del grupo El Paso: Antonio Saura- a quien conoce durante su estancia en París en 1958 y quien desde entonces se convierte en su gran amigo-, Manolo Millares, Gerardo Rueda, Gustavo Torner y Eusebio Sempere. Debido a este elenco de artistas que se reúnen en torno al Museo de Arte Abstracto Español de Cuenca, durante la década de los sesenta, algunos autores han denominado a este grupo, próximos a la corriente informalista, como "grupo de Cuenca"14 o "escuela de Cuenca"15. Sin embargo, Bonifacio Alfonso siempre se opuso a dicho encasillamiento al considerar que "allí no había grupo de pintores, había amigos... de escuela de Cuenca nada. [...] Había un grupo de artistas que venían, se iban... y algunos volvían a venir"1

Su primer grabado está fechado en 1968. Desde ese momento hasta la llegada de los setenta, realiza más de sesenta aguafuertes, para lo cual emplea planchas de cobre y de zinc. Estas últimas son las que más le gusta utilizar. Para adquirirlas, en lugar de acudir a las tiendas especializadas en materiales artísticos, va a las ferreterías donde venden piezas de zinc que se usan para realizar los trabajos de desagües de los tejados. Le gustan porque presentan

12 Por el taller -ubicado en la calle San Pedro de Cuenca- a pesar de que pasan unos cuantos artistas, entre otros Gabriel Ramos Uranga, el único que trabaja de manera constante es Bonifacio.

13 Entrevista.

4 Bajo el título El grupo de Cuenca, entre 1997 y 1998, se celebran tres exposiciones en la Sala de las Alhajas de la Caja de Madrid, Casa del Cordón de Burgos y Parque de la Ciudadela de Pamplona.

15 Para mayor información acerca de este tema se pueden consultar las siguientes publicaciones: BOZAL V.: "Grabado y obra gráfica en el siglo XX", Summa Artis. Historia General del Arte, Vol. XXXII, Madrid, Espasa-Calpe, 1978, pp. 394-395; DE LA TORRE, A y DE LUCAS RAMÍREZ, J.: La poética de Cuenca: 40 años después, 1964 2000 [Cat. Expl, Op. cit, DE LA TORRE A. :Pero hubo alguna vez un grupo de Cuenca?, Madrid Caja de Ahorros, 1998, pp. 55-73 o DE LA TORRE, A. "El grupo Cuenca" Guadalimar, Madrid, junio de 1998, p. 22

16 PÉREZ HERNANDO, R.: "Composición-descomposición-composición", Bonifacio (1970-1990). Madrid, Galería Rafael Pérez Hernando, 1998, p. 20 numerosas imperfecciones ${ }^{17}$. En numerosas ocasiones, coloca la plancha con barniz en el suelo de su taller, que está repleto de gravilla, para dañarlas aún más ${ }^{18}$.

A excepción de un grabado, que está estampado en Madrid por el Grupo Quince ${ }^{19}$, el resto los estampa y edita él mismo en el taller conquense de Antonio Lorenzo. Seguramente, debido a que se encuentra en pleno periodo de aprendizaje, comienza realizando tiradas muy cortas -de cinco, diez ejemplares- que posteriormente aumenta a veinte o veinticinco.

Con respecto a los papeles, utiliza principalmente el papel español Guarro, seguido del papel alemán y, sólo en uno ocasión, el papel japonés. A pesar de que el conquense Segundo Santos surte de papeles -que hace a mano- al museo para realizar las ediciones, a Bonifacio no le gusta emplear ese tipo de soporte de gran calidad porque considera que, de esa manera, se le otorga más valor al papel que al grabado en sí. A este respecto, él mismo comenta:

"[...] Segundo Santos era un hombre que vivía en Cuenca. Hacía un papel a mano de maravilla que le compraba todo Zóbel. Sin embargo, el papel que hacía Santos no me gustaba utilizarlo porque al ser un papel tan bueno el grabado perdía calidad, ya que el papel le ganaba al grabado. A mí lo que me gusta emplear es el papel Guarro corriente"20.

7 Por el contrario, el cobre, al tratarse de un metal más duro, no se estropea.

18 Entrevista..

19 El Grupo Quince se constituye, el 1 de octubre de 1971 en la madrileña calle Fortuny, no ${ }^{\circ}$. Está constituido por quince socios, de profesiones diversas (médicos, arquitectos, abogados, etc.), ajenos a mundo artístico pero afionados al arte que llevan a cabo la creación de un centro artístico dedeado a a la realización y prom de otras disciplinas de acercarse al mundo del grabado. Su actividad se desarrolla entre 1971 y 1985 a través de tres campos diferentes y complementarios: taller, galería y editorial. Se crea como un centro de difusión cultural, de experimentación e investigación de nuevas técnicas y de promoción del grabado, como un centro de referencia donde los artistas comparten sus experiencias; contribuyendo, así, a revitalizar el mundo de la gráfica que por este periodo se encuentra aletargado. Para un mayor conocimiento del tema se pueden consultar las siguientes publicaciones: GARRIDO, C.: "El Grupo Quince: pintores que graban y algo más", en LORENZO, A. et. al.: Grupo Quince (1972-1975). Colección privada Antonio Lorenzo Zaragoza. Diputación Provincial de Zaragoza 2006, pp. 15-19. GENER, M. "En CoAntonio Lor" Grupo Quince (1972-1975). Colección privada Antonio Lor

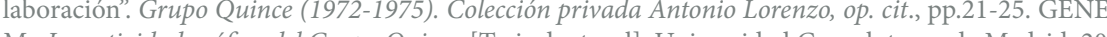
M.: La actividad gráfica del Grupo Quince [Tesis doctoral], Universidad Complutense de Madrid, 2013 o GALLEGO, A.: Historia del grabado en España. Madrid, Cuadernos de Arte Cátedra, 1990 
Por lo general, se trata de grabados en blanco y negro -aunque en contadas ocasiones también emplea color- que presentan formas abstractas. Ninguno de ellos está titulado, lo que acentúa aún más la idea de indeterminación. Nos encontramos ante una serie de formas planas que ocupan prácticamente la totalidad de la plancha. Observamos diferentes grosores de líneas -en las que predominan las curvas- que se entrelazan unas con otras originando un enmarañamiento de formas. A pesar de tratarse de formas abstractas, en ocasiones parece que nos encontremos contemplando una serie de estructuras rocosas. En este sentido y, como se hará más perceptible en el periodo siguiente, el artista se mueve entre la figuración y la abstracción, donde crea un estilo propio.

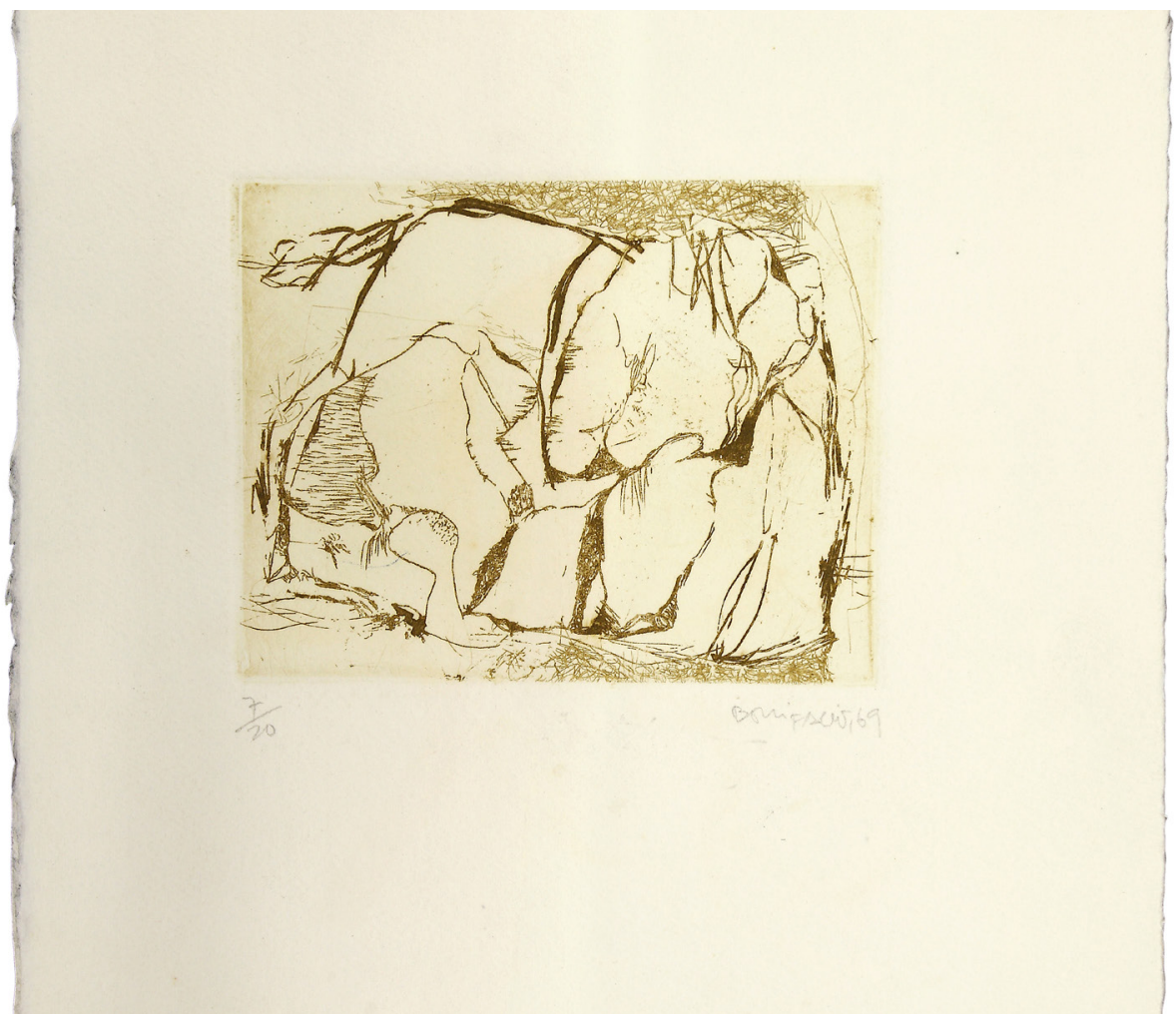

Fig. 1: Sin título. 1968. Grabado. Bonifacio Alfonso. (Museo de Bellas Artes de Bilbao: Bonifacio Alfonso. Obra gráfica completa 1968-1982. Museo de Bellas Artes de Bilbao, 1983, s. p.)
En la década posterior, se debe tener en cuenta que, desde 1970 -fecha en la que tiene lugar su primera exposición individual con la galería madrileña Juana Mordó y también el viaje a Avignon para visitar la muestra picassiana del Palais des Papes, sobre la que Rafael Alberti escribe un libro-, la galería tiene la exclusividad de toda su producción ${ }^{21}$.

Durante estos años abandona Cuenca temporalmente para acudir a diversos talleres de grabado. En 1973 viaja a Barcelona para trabajar, durante tres meses, en la Editorial Gustavo Gili, donde coincide con Joan Miró. Después de esta estancia regresa como maestro litógrafo ${ }^{22}$. En 1975 acude al taller madrileño del Grupo Quince para trabajar junto al litógrafo Don Herbert. Y en 1978 se traslada a la capital francesa para estampar con el danés Peter Bramsem en el prestigioso taller $\mathrm{Clot}^{23}$, donde coincide con el miembro del grupo Cobra, Pierre Alechinsky ${ }^{24}$. A partir de ese año, acude al taller de grabado que se pone en funcionamiento en el Museo Provincial de Cuenca ${ }^{25}$. Por otro lado, se debe tener en cuenta que desde 1969 y, en toda la década posterior, una de los centros de difusión artística que respaldan su producción gráfica es la Galería vizcaína Mikeldi, dirigida por Gotzone Echevarría. Dicho espacio, abierto de 1964 a 1980, se convierte en un lugar de encuentro donde se debate sobre arte, cultura y política, se hacen presentaciones de libros y se realizan recitales de poesía ${ }^{26}$. Se trata de una de las pocas galerías vizcaínas junto con Aritza que apuestan por el arte gráfico.

21 BONET, J. M: "Bonifacio o el combate por la expresión", Bonifacio en los campos de batalla. Madrid, Ediciones Exposición, 2007, p. 12

22 Entrevista.

23 Peter Bramsem se convierte en 1963 en el litógrafo del prestigioso Taller Clot de París. Dicho taller es fundado en 1896 por August Clot considerado como el mejor litógrafo de París. Sus habilidades técnicas para trabajar con distintos colores llaman la atención de jóvenes artistas como Edgar Degas, Henri de Toulouse-Lautrec, Paul Cézanne y Auguste Rodin. En 1934 se pone al frente del oficio su hijo August, durante esta etapa trabajan en el taller reconocidos artistas como Henri Matisse, Armand Guillaumin y Tsuguharo Foujita. Con la llegada de Peter Bramsem, en 1963, acuden a trabajar allí artistas como Asger Jorn, Pierre Alechinsky, Bram van Velde, Bengt Lindström y Henri Michaux, entre otros. Información extraída del INSTITUTO NACIONAL DE LA ESTAMPA, MUSEO NACIONAL DE LA ESTAMPA: Atelier Clot, Bramsem \& George, http://www.cultura.gob.mx/micrositios/atelir_clot/ (consultado el 28/02/2016)

24 BONET, J.M.: "Bonifacio o el combate por la expresión", Bonifacio en..., op. cit., p. 14

25 Por él pasaron diversos pintores que comenzaron su andadura en el mundo del grabado durante finales de los setenta y principios de los ochenta. En él impartieron clases de grabado profesores como Julián Pacheco o Paolo Fiaccarini, procendente de Stampería Santa Chiara, en la ciudad italiana de Urbino, uno de los centros más relevantes en estampación. Información extraída de MUÑOZ MARQUINA, A "El arte de las dos últimas décadas del siglo XX en Cuenca", Añil, 21, pp. 31-34.

26 A dichas tertulias acuden, entre otros, los poetas Luciano Rincón, Vidal de Nicolás, Sabina de la Cruz y 


\section{DeSARROLlo de SU ObRA GRÁfica. DÉCAdA de los SETENTA}

En esta época, hace cerca de 150 grabados. El año 1972 es especialmente prolífico. Realiza, fundamentalmente aguafuertes -que en el caso de las series, a excepción del libro Tomilleros (1979), combina con aguatinta- y, en menor medida, serigrafías y litografías.

Con respecto a los aguafuertes emplea planchas de zinc y, particularmente, de cobre. Por lo general, tan sólo utiliza el color blanco y negro. Por el contrario, las serigrafías y litografías se llenan de vivos colores, llega a introducir hasta seis tonalidades. El propio artista reconoce que, aunque no le gustan los grabados en color, de vez en cuando incorpora un poco para satisfacer el gusto del público. Ahora bien, en el caso de las litografías es diferente porque para él es como si estuviese haciendo acuarelas ${ }^{27}$.

Por otro lado, siempre que se enfrenta a la plancha vacía realiza un boceto previo. En ocasiones ha empleado un gouache para, posteriormente, traspasarlo a la plancha. Siempre realiza este proceso de manera directa, sin calca, puesto que lo que le interesa mostrar no es una obra perfecta, sino exponer también los fallos que se han ido produciendo en el largo proceso de trabajo porque, como él mismo reconoce, lo que más le gusta del grabado es la grata sensación que le produce el no saber nunca lo que va a suceder ${ }^{28}$.

En los aguafuertes, por lo general, utiliza el papel español Guarro. Como excepción encontramos el libro Serán cenizas (1978) - en el que a pesar de que los textos se componen sobre papel Súper Alfa (Casa Guarro), los grabados se estampan sobre papel Velín-D' Arches-; uno sin titular (1971) y un Insecto (1972) -en los que usa el papel hecho a mano obtenido en el taller conquense de Segundo Santos-; la serie Insectos (1972) -en los que

Blas de Otero, entre otros. Todos ellos muy relacionados con el movimiento de Estampa Popular de Vizcaya, cuyos grabados se exponen y venden a precios muy asequibles. Durante los primeros años atiende a la pintura anterior a la Guerra Civil, mientras que en los siguientes se interesa por los artistas más destacados del momento. Entre los que muestran sus trabajos, además de Bonifacio Alfonso, destacan los artistas pertenecientes o próximos al grupo de Estampa Popular (Agustín Ibarrola, María Francisca Dapena, José Ortega o Dionisio Blanco) y aquellos artistas integrantes del Movimiento de Escuel Vasca (Mendiburu, Zumeta, Balerdi, Ortiz de Elguea, etc.) junto a jóvenes promesas (Txopitea, Begoñ del Valle, Nagel, Moruillas, Mirantes, Santos Iñuriete etc). Información extraída de FERNÁNDEZ

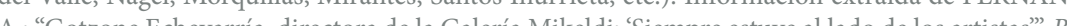
A.: “'GotzoneEchevaria, directoa de $n^{\circ} 182$, Bilbao, mayo de 2004, p. 16

27 Entrevista...

28 Ibid usa el papel francés Rives- y algunas litografías en las emplea el papel francés Arches. En relación a la etapa anterior, a la hora de realizar aguafuertes -técnica que ya dominasus tiradas aumentan (por lo general, 50), llegando en las serigrafías hasta los 75 o 100 ejemplares ${ }^{29}$.

Durante los primeros años de la década, se encarga de estampar y editar sus propios grabados. Sin embargo, al poco tiempo descubre que este trabajo tan mecánico le aburre. Sin embargo, disfruta haciendo las Pruebas de $\mathrm{Artista}^{30}$. Por ese motivo, en la serie de aguafuertes denominados Insectos (1972) observamos cómo de los 50 ejemplares de los que se compone, Bonifacio estampa del 1 al 10, mientras que Pancho Ortuño -uno de los artistas que trabajan en la edición de obra gráfica del Museo de Arte Abstracto Español ${ }^{31}$ -estampa el resto. Del mismo modo, años más tarde, en 1978, nos encontramos con una felicitación de navidad estampada por el Grupo Quince y con otro grabado estampado por Antonio Machón, director de la galería vallisoletana Carmen Durango. Igualmente, la mayoría de las serigrafías -excepto una, fechada en 1977, que reproduce Javier Cebrián y Ángel Cruz- las estampa Abel Martín, el encargado de las estampaciones serigráficas del museo. A su vez, cuando se trata de litografías, las primeras las estampa su maestro en aquel entonces (1975), Don Herbert; mientras que las llevadas a cabo entre 1977 y 1979 -momento en el que acude al taller parisino Clot- las estampa el litógrafo Peter Bramsen.

La gran mayoría los edita la Galería Juana Mordó y él mismo. Por otro lado, cuando se traslada a otros talleres son -como ocurre con la estampación- o bien son sus propios maestros, o bien las galerías con las que éstos trabajan quienes estampan sus obras. De este modo, a raíz de su estancia en el taller barcelonés de Gustavo Gili, en 1974, nos encontramos con una serigrafía editada por el maestro catalán; o, entre 1977 y 1979 las litografías las edita el francés Yves Rivière ${ }^{32}$.

En cuanto a los libros, Cuatro orejas y rabo (1973) se convierte en el único que edita el Museo de Arte Abstracto Español; Norberto el Pata y Pitín. (1975) lo edita Gustavo Gil

29 MUSEO DE BELLAS ARTES DE BILBAO.: Bonifacio Alfonso. Obra gráfica completa. 1968-1982, Bilbao, Museo de Bellas Artes de Bilbao, 1983. Entrevista...

30 Junto a él también pasaron por Cuenca, entre otros, los hermanos Blassi, Ricard Giralt-Miracle Charo

31 Mirat, Meli Pérez Madero, Joaquín Sáenz, Manolo Valdés, Mitso Miura, Nacho Criado, Cristóbal Hara, Javier Cebrián, Ángel Cruz, Salvador Victoria, Fernando Nuño, Amadeo Gabino, etc.

32 Yves Rivière (1930-2008) fue un prestigioso editor de libros de arte de artistas como Man Ray. 
En sus grabados alterna la forma figurativa y la abstracta. Plasma la realidad que le rodea de manera intuitiva, es decir, sin ideas preconcebidas. Se trata de una obra gestual, automática y muy dinámica en la que se aprecia la rapidez en el trazo. Su obra se sitúa, por tanto, dentro de la órbita del expresionismo abstracto del grupo Cobra y, en cierta manera, también del surrealismo. Presenta afinidades con artistas como Pierre Alechinsky, Asger Jorn, Willen De Kooning y Roberto Matta. A todos ellos, salvo a De Kooning los llega a conocer, con Alechinsky coincide en París en el taller de Peter Bramsem; en Cuenca, invitado por Antonio Saura, conoce a Asger Jorn y con Roberto Matta llega a establecer una estrecha $\operatorname{amistad}^{33}$.

Algunos autores como Antonio Saura perciben en la obra de Bonifacio un halo de sensualidad y de deseo que, a partir de los últimos años de la década de los sesenta, inunda toda su producción. Hasta el punto de afirmar que "tanto la ambigüedad de las formas como la interrelación de los elementos lo convierten en uno de los pocos ejemplos válidos del erotismo pictórico actual" ${ }^{34}$.

En su obra se produce un contraste. De una parte, de manera meticulosa nos muestra el mundo, fácilmente reconocible, de los insectos, solos o en grupo; y de otra, nos presenta una obra más difícil de identificar que, a menudo, desarrolla en viñetas, en la que nos enseña, a la manera de la metamorfosis kafkiana ${ }^{35}$, seres deformados con cierta apariencia humana en continua transformación.

Durante estos años, en Cuenca encuentra su refugio idóneo para concentrarse en sus grandes pasiones, el grabado y la pintura de un lado, y por el otro los toros y los insectos. Estas dos últimas y especialmente los insectos se convierten en el tema central de algunos de sus grabados.

33 BONET, J. M.: "Bonifacio o el combate por la expresión", Bonifacio en los campos de batalla. Op. cit., p. 14.

34 SAURA, A.: Bonifacio Alfonso. Óleos, grabados y dibujos. Durango, Salas Municipales de Cultura de Durango, 1971, s. p.

35 BOZAL, V:: "Grabado y obra gráfica en el siglo XX”, op. cit., p. 778.

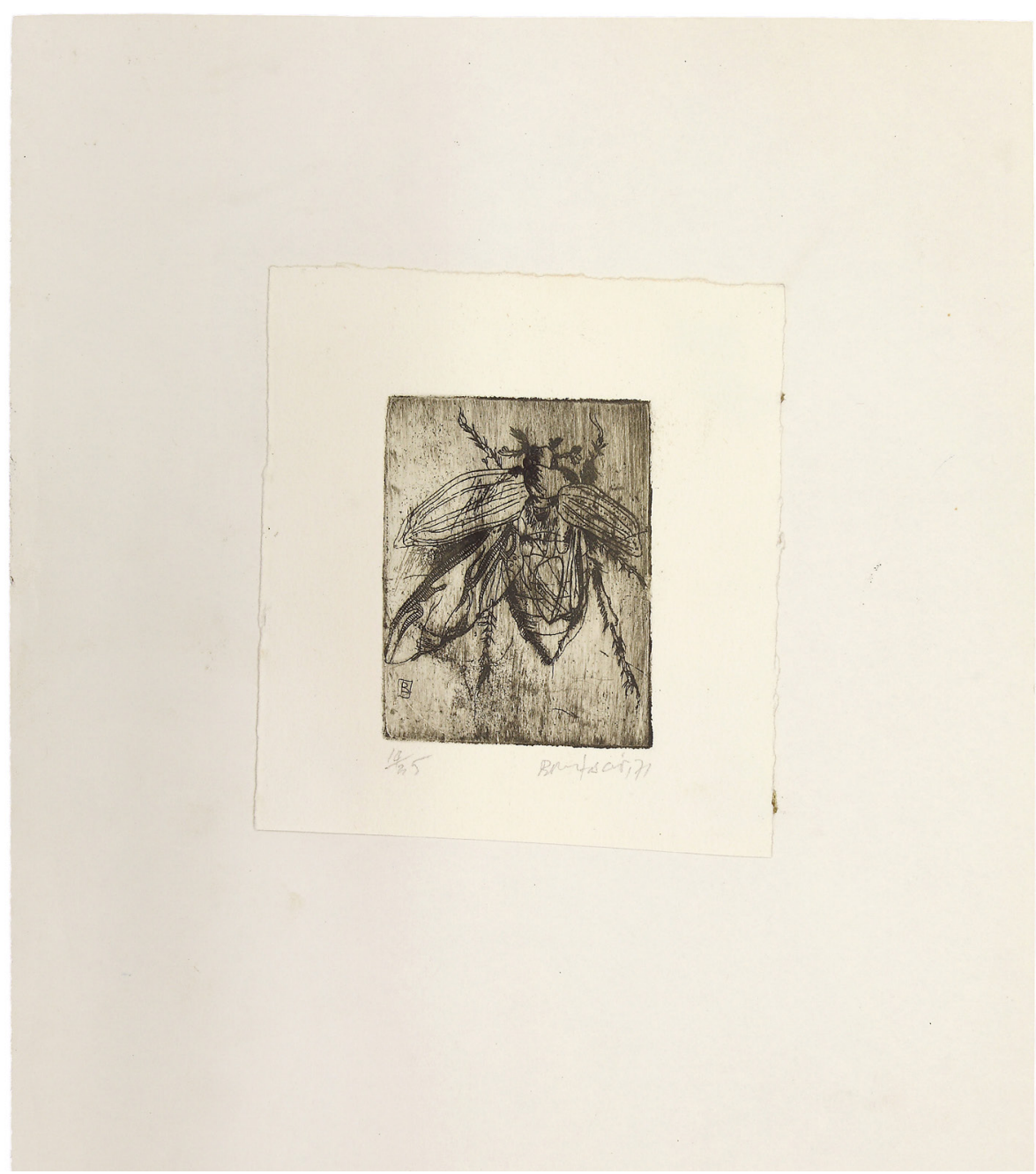

Fig. 2: Insectos. 1971. Aguafuerte. Bonifacio Alfonso. (Museo de Bellas Artes de Bilbao: Bonifacio Alfonso. Obra gráfica completa 1968-1982. Museo de Bellas Artes de Bilbao, 1983, s. p.) 


\section{MOTIVOS DE SU PRODUCCIÓN}

En 1971 realiza el primero de una larga lista de aguafuertes sobre cobre y zinc en los que, como si de un entomólogo se tratara, recrea ese mundo peculiar. En 1972, lleva a cabo un conjunto que la galerista Juana Mordó edita en sucesivas series. Realiza un total de cuarenta y ocho planchas de las cuales Bonifacio estampa del 1 al 10 y Pancho Ortuño del 10 al 50. Simultáneamente, realiza numerosos dibujos a lápiz de carácter naturalista, relativo a plantas, animales y, especialmente, insectos ${ }^{36}$. Este motivo se convierte en una especie de obsesión que le lleva a coleccionar, como si de un estudioso en la materia se tratase, bichos que recoge en la orilla de río Júcar a donde, durante 28 años, acude junto a su amigo José Morante, quien le enseña el arte de pescar a la mosca. Una vez capturados, los que no emplea como cebo para la pesca, los mete, de manera ordenada, en cajas. En concreto, para pescar truchas, recoge unos gusanos blancos que cría en los armarios de su casa. Los recopila en cajas ordenadas alfabéticamente, pegados en libros o prensados entre dos vidrios ${ }^{37}$

Esta fascinación está relacionada con el acceso que, gracias a Zóbel, tiene a las estampas originales del grabador e ilustrador norteamericano Leonard Baskin ${ }^{38}$, quien realiza numerosas obras de animales, y también de insectos ${ }^{39}$. Es precisamente con esta última serie con la que el artista guipuzcoano queda cautivado. En su casa se dedica a observarlos detenidamente hasta que un día decide copiar uno sobre un escarabajo ${ }^{40}$. Posteriorment comienza a realizar sus propias obras. Puede afirmarse, por tanto, que Baskin se convierte en uno de sus referentes. Por otro lado, algunos autores ${ }^{41}$ han apreciado una similitud con las litografías del inglés Graham Vivian Sutherland. Aunque también se puede reconoce una cierta influencia de los grabados de la misma temática, denominados Extraños insectos, del pintor grabador belga James Ensor.

36 Posteriormente, continúa cultivando esta afición por la entomología en cuadros como La mesa de los insectos (1991) o Insectos y una muela (1996)

37 Entrevista...

38 El grabador e ilustrador estadounidense Leonard Baskin (1922-2000) es uno de los referentes de Bonifacio Alfonso. Se inspira en sus grabados de búhos, pájaros y demás insectos para realizar su propia serie de insectos que lleva a cabo en 1972. Entrevista a Bonifacio Alfonso, San Sebastián, 7 de enero de 2009

39 BONET, J. M.: "Bonifacio o el combate por la expresión", Bonifacio en .... op. cit., p. 12

40 Entrevista...

41 DE BARAÑANO, K. Ma; GONZÁLEZ DE DURANA, J. y JUARISTI, J.: Arte en el País Vasco. Madrid, Cuaderno Arte Cátedra, 1987, p. 391
Los grabados con este asunto los realiza a gran escala. Los primeros aguafuertes son realistas, las formas son claramente identificables (moscas, mosquitos, libélulas, escarabajos, saltamontes, arañas, hormigas, avispas, ciempiés, abejas, etc.). Parece como si el artista se convirtiera en un estudioso de cada insecto, reconstruyendo el mecanismo de cada uno de ellos ${ }^{42}$. Plasma la morfología externa, las peculiaridades y los complicados esquemas de cada insecto ${ }^{43}$. En ocasiones, representa la trasformación de una larva en una mariposa o el instante en el que un escarabajo expulsa sus huevas.

Por lo general, representa un solo insecto ocupando toda la imagen, de frente o de lado. Emplea diferentes formatos (rectangular, cuadrangular o circular) y tamaños. Ha llegado a realizar grabados de la dimensión de un sello que, después, ha empleado para pegar en los sobres de las cartas que envía a sus amigos ${ }^{44}$. Posteriormente, las formas van perdiendo nitidez, hasta el punto de que cada vez es más difícil identificar la familia que nos muestra. Por lo general, esta peculiaridad la pone de manifiesto cuando representa multitudes, donde observamos una especie de clan desorganizado en medio de un caos espacial ${ }^{45}$.

De analizar, con cierto rigor científico, la evolución de una nueva especie de insectos, pasa a sumergirse en una ensoñación surrealista donde recrea un mundo repleto de seres fantásticos con apariencia grotesca. Se trata de unas composiciones geométricas que dan lugar a la aparición de unas formas abstractas mitad hombres, mitad monstruos. Por ejemplo, en 1972 nos encontramos ante un grabado, editado y estampado por él mismo, en el que muestra a un extraño ser de grandes dimensiones. Se trata de una especie de humanoide desnudo cuyo rostro, representado por medio de una gran mancha, no podemos apreciar. A su vez, dota al cuerpo, asimétrico y descompuesto, de una poderosa musculatura. El carácter volumétrico de las formas nos recuerda más a una escultura que a un dibujo.

42 RUIZ QUINTANO, I.: Bonifacio, op. cit., p. 61

43 BORRAS, P.: "Bonifacio. Pintar la luz, oficio de tinieblas", Bonifacio en los campos de batalla, op. cit., p. 23

44 Entrevista...

45 BORRAS, P.: "Bonifacio. Pintar la luz, oficio de tinieblas", Bonifacio en los campos de batalla, op. cit., p. 
Años más tarde, en 1976, realiza un grabado muy similar. A pesar de que en esta ocasión sintetiza las formas, mantiene la esencia del pseudo retrato de cuerpo desnudo. Aunque continuamos sin poder identificar el rostro, apreciamos sus extremidades, el tronco y sus órganos sexuales. Esta obra nos recuerda a la serie de Mujeres que Willem De Kooning desarrolló en los años 50, en la cual plasma de manera grotesca el cuerpo femenino, realzando los rasgos sexuales entre un caos de violentos brochazos de color (Fig. 3).

De la misma manera, en la serie de seis serigrafías de intensos colores denominada Sorginak (1972), que estampa Abel Martín y edita Juan Mordó, en dos de ellas a través del enmarañamiento de líneas se insinúa la presencia de seres desfigurados. En una observamos el retrato, caricaturizado, de un hombre realizado de perfil; mientras que en otra aparece un espécimen de cuya cabeza le sale un brazo. La obra está inundada de grandes manchas de vivos colores (seis). En algunas ocasiones, influenciado por el action painting, deja chorrear la pintura por la plancha.

Por otro lado, comienza a utilizar un recurso que empleará, a partir de 1975, con cierta asiduidad ${ }^{46}$. Se trata de las tiras de cómic en las que continúa mostrándonos seres deformados. Recrea un mundo onírico repleto de formas surrealistas en el que la identificación de las mismas resulta una tarea complicada. Por ejemplo, en la litografía, fechada en 1975 y estampada por Don Herbert, nos encontramos con una serie de dibujos semi-abstractos, en blanco y negro, enmarcados en doce cuadrados. A pesar de que en las primeras viñetas prácticamente no podemos identificar las formas -a excepción de una cabeza de toro colgada de una pared, o la presencia de una especie de puño en alto-según avanzamos, éstas se vuelven más nítidas. Hasta el punto que en algunas de ellas -en las tres centrales- las líneas comienzan a configurar la imagen del dictador Franco cuya cara, como si de una figura de cera se tratase, se está derritiendo. En la penúltima viñeta retrata al dictador vestido con el uniforme de gala. En esta litografía encontramos cierta relación con la obra el Sueño y mentira de Franco ${ }^{47}$ de Pablo Ruiz Picasso ${ }^{48}$. Parece como si, al igual que hiciera el artista andaluz, Bonifacio tratara de denunciar la complicada situación por la que atraviesa España, en este caso, a punto de fallecer el Dictador.

46 A pesar de que la primera vez que lo emplea es en un grabado de 1971, no será hasta mitad de la década de los setenta cuando lo utilice de manera más constante.

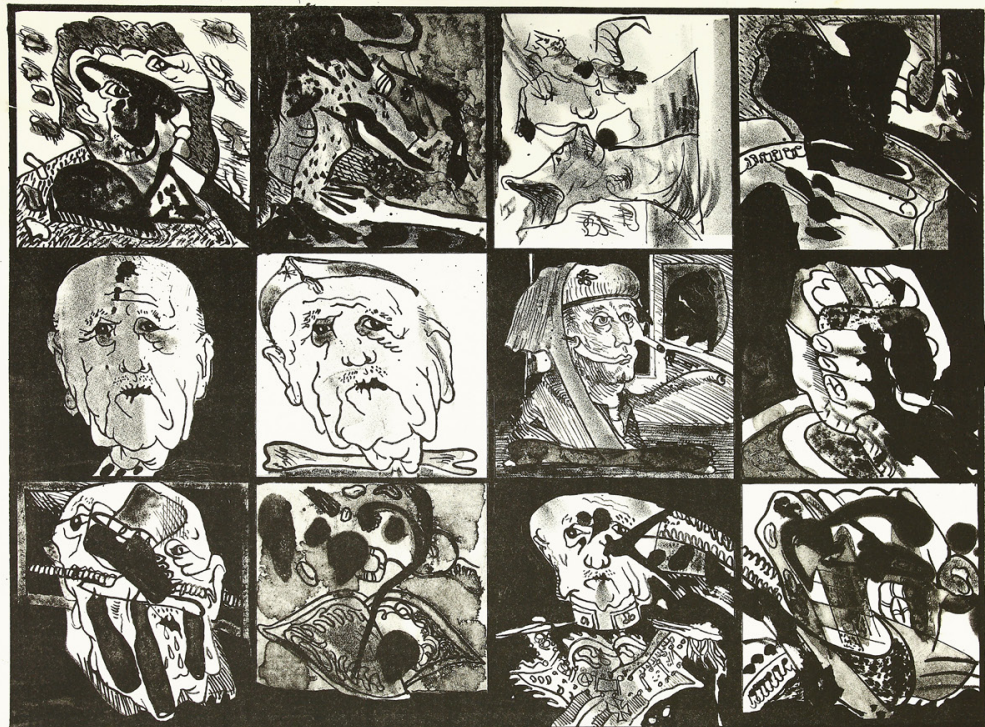

Sin titulo. 1975. Litografía. Aguafuerte. Bonifacio Alfonso. (Museo de Bellas Artes de Bilbao: Bonifacio Alfonso. Obra gráfica completa 1968-1982. Museo de Bellas Artes de Bilbao, 1983, s. p.)

Este procedimiento también lo utiliza en la mayoría de los libros de bibliofilia. Como ya hemos mencionado anteriormente, en 1973 se publica su primer libro, el único que edita el Museo de Arte Abstracto Español. Lo diseña conjuntamente con los hermanos Blassi y en él recoge sus impresiones taurinas. Previamente realiza numerosos dibujos preparatorios con lápiz y tinta china. Emplea frases, expresiones y giros de diversos toreros, críticos taurinos

47 Entre el 8 y 9 de enero de 1937, Picasso escribe el texto del folleto Sueño y mentira de Franco, al que acompaña una serie de 18 aguafuertes con aguatinta. Estas obras fueron un encargo del Gobierno de la República española para la Bxposicion Interta República spanta el Guernica, en el que plasma el bombardeo sufrido por esa villa el 26 de abril de 1937. Al igual que posteriormente realiza el artista donostiarra, Picasso divide la imagen en viñetas, en este caso en 9. 48 BOZAL, V.: "Grabado y obra gráfica en el siglo XX", op. cit., p. 778 
y escritores a los que dedica su personal faena (Eugenio Noel, Gregorio Corrochano, Rafae Alberti, José María de Cossío, José Bergamín). Fernando Zóbel se refiere a este trabajo del siguiente modo:

“[...] No me acuerdo muy bien de cómo surgió la idea del libro. Lo que sé es que desde el primer momento nos pareció extraordinariamente interesante la expresión gráfica del arte taurino por un pintor de categoría que antes había sido un torero de verdad"49.

Otro ejemplo lo encontramos en el libro Norberto el Pata y Pitín. (1975) para su realización lleva a cabo una serie de cinco aguafuertes y aguatinta, en blanco y negro, sobre plancha de cobre. Se trata de una historieta, narrada en forma de cómic -la plancha está dividida en recuadros- y los personajes - una serie de monigotes deformados, de apariencia grotescaaparecen hablando entre ellos, por medio de bocadillos, sobre las centrales nucleares ${ }^{50}$. La edita Gustavo Gili dentro de la colección Las Estampas de la Cometa y la estampa Alberto Coscolla.

Un año después, volvemos a toparnos con una serie de figuras deformadas, inmersas en un ambiente onírico y misterioso. Se trata del libro Sopas y Manjares (1976) publicado por la editorial francesa Yves Rivière (Fig. 5). De los quince aguafuertes, en blanco y negro, que lo componen, once aparecen representados en forma de viñetas -divide la imagen en 4,9y 16 recuadros-. El portafolio está basado en el texto del libro de recetas de cocina del clásico de la gastronomía española, Ruperto de Nola -Libro de manjares, guisos y potajes (impreso en el siglo XVI ${ }^{51}$.

A finales de la década de los setenta, coincidiendo con su estancia en el taller parisino de Clot Bramsen, sus grabados evolucionan de unos dibujos de un marcado expresionismo y una gran acción y fuerza, a unas formas más infantiloides que abarrotan toda la estampa. A partir de ahora, las formas se vuelven más espaciadas, más aéreas, más luminosas, repletas

49 RUIZ QUINTANO, I., Bonifacio, op. cit., p. 30.

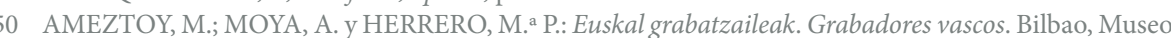
de Bellas Artes de Bilbao, Caja de Ahorros Vizcaína, 1979, p. 28.

51 El artista disfruta realizando este trabajo, ya que, como buen donostiarra, entre sus aficiones se encuentra el gusto por la gastronomía. Además, durante este tiempo, su mujer (M. ${ }^{a}$ Carmen Flores) dirige el Mesón Flores de las Casas Colgadas de Cuenca. de salvajes colores y de un fuerte lirismo ${ }^{52}$. Un ejemplo de ello lo encontramos en las litografías que estampan Clot y George Bramsen y edita Yves Rivière. Alguna de ellas, aparecen tituladas (Emen, Ánimas, Bazar, Triana, Semáforos). Sin embargo, estos títulos no hacen más que acentuar el carácter ambiguo de las formas, ya que al tratarse de formas abstractas la identificación de las mismas se hace prácticamente imposible.

Prosiguiendo con los libros de bibliofilia, y dejando a un lado la disposición de sus seres oníricos en tiras de cómic, nos encontramos con el portafolio Serán cenizas (1978) editado por Antonio Machón, director de la Galería Carmen Durango. El álbum, que corresponde a la tercera monografía de la colección Marzales, contiene cinco aguafuertes originales de Bonifacio y cinco sonetos inéditos del escrito, ensayista y poeta madrileño José Bergamín. En esta ocasión los seres fantásticos revolotean por todo el espacio en una ausencia total de perspectiva.

Por último, en 1979 realiza el libro Tomilleros. Se trata de 115 serigrafías estampadas en os Talleres de artes gráficas Gasaló (Valencia) y editadas por Antonio Pérez, autor de los textos y director de la editorial Antojos ${ }^{53}$. El título, Tomilleros, alude al nombre que reciben en Cuenca los voyeurs. Las extrañas figuras - no se distingue bien si nos encontramos ante formas vegetales, animales o humanas- aparecen distanciadas unas de otras, como si estuvieran danzando en el espacio. Ha desaparecido el abigarramiento de imágenes anteriores. Por otro lado, las cuatro primeras se han llenado de llamativos colores lo que acentúa el carácter aniñado de su obra. De esta manera, su obra se acerca cada vez a la imaginería propia del movimiento surrealista, plagado de seres fantásticos, quiméricos, caricaturescos, etc.

Finalmente, una vez que abandona Cuenca y establece su residencia en Madrid, en torno a 1996, las litografías las estampa Antonio Gayo, discípulo de Don Herbert, al que el artista guipuzcoano dedica las siguientes palabras: “Tengo la suerte de que mi vecino y amigo es Antonio Gayo, que es un maestro de la litografía en piedra. Creo que en España hay muy pocos talleres que trabajen la litografía tradicional sobre piedra" ${ }^{\prime 54}$.

52 BONET, J. M.: "Bonifacio o el combate por la expresión", Bonifacio en los campos de batalla, op. cit., p. 16.

53 En esta editorial, creada en 1978 por Antonio Pérez, además de Bonifacio Alfonso participan artistas como Antonio Saura, A. Ràfols Casamada, Pío Baroja, Guinovart, Equipo Crónica, etc.

54 HARGUINDEY, Á.: "La pintura también deja cicatrices. Entrevista con Bonifacio", Bonifacio en lo campos de batalla, op. cit., p. 235. 


\section{CONCLUSIONES}

La incursión de Bonifacio Alfonso en el grabado a finales de los años sesenta se produce gracias a la amistad que establece con el mecenas de arte y fundador del Museo de Arte Abstracto Español de Cuenca, Fernando Zóbel, quien le brinda la oportunidad de adentrarse en esta disciplina muy díficil de llevar a cabo en su localidad de origen, debido a la prácticamente inexistencia de talleres para tal fin. Por este motivo, para probar suerte en el grabado debe buscar fuera talleres tanto nacionales como extranjeros que cuenten con equipos de calidad, con maestros-estampadores especializados en cada uno de los procedimientos, poniendo a su disposición tanto los materiales y herramientas como los conocimientos necesarios para su dominio. En cuanto a talleres nacionales acude a Cuenca, ciudad en la que se instala durante más de 20 años y en la que aprende la técnica del grabado calcográfico en el taller de Antonio Lorenzo, creado por iniciativa de Zóbel para trabajar en verano a la sombra del Museo de Arte Abstracto Español. Durante breves estancias de tiempo abandona la ciudad de La Hoz del Júcar para continuar instruyéndose en dicha disciplina. Aprende la técnica litográfica en la editorial barcelonesa Gustavo Gili (1973), la cual la perfecciona de la mano del estadounidense Don Herbert en el madrileño taller del Grupo Quince (1975). A nivel internacional, en 1978 acude al taller parisino Clot para perfeccionar la técnica con el prestigioso litógrafo Peter Bramsen.

Desde que realiza su primer grabado en 1968 hasta finales de los setenta realiza alrededor 210 grabados. Fundamentalmente aguafuertes y, en menor medida, serigrafías y litografías. También lleva a cabo diversos libros de bibliofilia.

En su proceso de trabajo primera realiza un boceto que de manera directa traspasa a la plancha para poder dejar constancia también de los fallos cometidos en el proceso de elaboración de la estampa. Con respecto a los papeles, además de emplear el papel artesanal realizado por Segundo Santos, en los aguafuertes utiliza, normalmente, el papel español Guarro y en ocasiones el francés Rives o el Arches para alguna litografía.

Por otro lado, hemos podido constatar cómo a pesar de que al principio se encarga él mismo de estampar y editar sus propios grabados, lo que viene a evidenciar que se trata de un auténtico grabador que participa de todo el proceso de trabajo, posteriormente serán otros profesionales los encargados de estamparlos. En cuanto a la edición la mayoría, a excepción de aquellos que realiza en otros talleres, los edita él mismo y la Galería Juana Mordó.
Con respecto a la forma podemos afirmar que en sus grabados alterna la figuración y la abstracción. Algunos autores lo incluyen dentro de la denominada Escuela de Cuencaaunque el propio artista se oponga a dicho encasillamiento- formada por artistas cercanos a la corriente informalista, sin embargo, la pluralidad de fuentes de la que bebe hace que también se sitúe dentro de la esfera surrealista y, particularmente, del expresionismo. Sus grabados, al igual que el resto de su producción pictórica, destilan humor e ironía. En su obra se pueden reconocer insectos, personales y objetos. Figuras deformadas e indescriptibles en ocasiones, en otras, como en sus primeros insectos, claramente reconocibles que revolotean y se mueven incansablemente por todo el espacio.

De todo lo expuesto se deduce que Bonifacio Alfonso es un auténtico artista-grabador que domina las técnicas de estampación y que se involucra en todo el proceso creativo, lo cual le valdrá en 1993 el Premio Nacional de Grabado otorgado por la Calcografía Nacional. 


\section{BIBLIOGRAFÍA}

AMEZTOY, M.; MOYA, A. y HERRERO, M. a P.: Euskal grabatzaileak. Grabadores vascos. Bilbao, Museo de Bellas Artes de Bilbao, Caja de Ahorros Vizcaína, 1979.

ARÓSTEGUI BARBIER, J. de: La pintura vizcaína de la posguerra. Del Grupo del Suizo a la Asociación Artística Vizcaína, Bilbao, La Gran Enciclopedia Vasca, 1972.

ARRIBAS, M. J.: 40 años de Arte Vasco (1937-1977). Historia y documentos, San Sebastián, Erein, 1979.

BARAÑANO, K. M. a de; GONZÁLEZ de DURANA, J. y JUARISTI, J.: Arte en el País Vasco. Madrid, Cuaderno Arte Cátedra, 1987.

BONET, J. M.: "Bonifacio o el combate por la expresión”, Bonifacio en los campos de batalla. Madrid, Ediciones Exposición, 2007.

BOZAL, V.: “Grabado y obra gráfica en el siglo XX”, Summa Artis. Historia General del Arte, Vol. XXXII, Madrid, Espasa-Calpe, 1978.

FERNÁNDEZ, A.: "Gotzone Echevarría, directora de la Galería Mikeldi: 'Siempre estuve al lado de los artistas"', Bilbao, no 182, Bilbao, mayo de 2004, p. 16.

GALLEGO, A.: Historia del grabado en España. Madrid, Cuadernos de Arte Cátedra, 1990

GARRIDO, C.: "El Grupo Quince: pintores que graban y algo más”, en LORENZO, A. et al.: Grupo Quince (1972-1975). Colección privada Antonio Lorenzo, Zaragoza, Diputación Provincial de Zaragoza, 2006, pp. 15-19.

GENER, M.: "En Colaboración". Grupo Quince (1972-1975). Colección privada Antonio Lorenzo, Zaragoza, Diputación Provincial de Zaragoza, 2006, pp.21-25.

GENER, M.: La actividad gráfica del Grupo Quince [Tesis doctoral], Universidad Complutense de Madrid, 2013.

GUASCH A. M.a: Arte e ideología en el País Vasco: 1940-1980, Madrid, Akal, 1985.
INSTITUTO NACIONAL DE LA ESTAMPA, MUSEO NACIONAL DE LA ESTAMPA: Atelier Clot, Bramsem \& George, http://www.cultura.gob.mx/micrositios/atelir_clot/ (consultado el 28/02/2016).

MACAZAGA LANAS, L.: "Talleres de grabado en el País Vasco durante el tardo franquismo y la transición”, Aacadigital, n 23, 2013. https://dialnet.unirioja.es/servlet/ articulo? codigo $=4530301$ (consultado el 20/02/2016)

MACAZAGA LANAS, L.: "El difícil panorama artístico al que debieron hacer frente los grabadores vascos durante el tardofranquismo y la transición", Aacadigital, n 27, 2014 http://www.aacadigital.com/contenido.php?idarticulo=974 (consultado el 21/02/2016).

MUÑOZ MARQUINA, A.: "El arte de las dos últimas décadas del siglo XX en Cuenca", Añil, 21, pp. 31-34.

MUSEO DE ARTE ESPAÑOL DE CUENCA: Colección de Arte Abstracto Español. Casas Colgadas. Museo Cuenca [Cat. Exp.], Museo de Arte Abstracto Español de Cuenca, 1966.

MUSEO DE BELLAS ARTES DE BILBAO.: Bonifacio Alfonso. Obra gráfica completa. 19681982, Bilbao, Museo de Bellas Artes de Bilbao, 1983.

ORTUÑO, P.: "Crece el Museo de Arte Abstracto de Cuenca", Guadalimar, no 35, Madrid, 1978, pp. 32-34.

PÉREZ MADERO, R.: Zóbel. Obra gráfica completa, Diputación Provincial de Cuenca Departamento de Cultura, 1999.

PÉREZ HERNANDO, R.: “Composición-descomposición-composición”, Bonifacio (19701990). Madrid, Galería Rafael Pérez Hernando, 1998, p. 20.

RUIZ QUINTANO, I.: Bonifacio. Madrid, Ediciones Turner, 1992.

SAURA, A.: Bonifacio Alfonso. Óleos, grabados y dibujos. Durango, Salas Municipales de Cultura de Durango, 1971, s. p.

TORRE, A. de la y LUCAS RAMÍREZ, J. de: La poética de Cuenca: 40 años después, 19642000 [Cat. Exp.], Centro Cultural de la Villa, Ayuntamiento de Madrid, 2004. 
TORRE, A. de la: ¿Pero hubo alguna vez un grupo de Cuenca?, Madrid, Caja de Ahorros 1998.

TORRE, A. de la: “El grupo Cuenca”, Guadalimar, Madrid, junio de 1998, p. 22.

ZÓBEL, F.: “Debe acabar la incertidumbre laboral del artista español”, El País, Madrid, 8 de marzo, 1978. 